\title{
Clavulanic acid production by Streptomyces clavuligerus: biogenesis, regulation and strain improvement
}

\begin{abstract}
Ashish Paradkar
Clavulanic acid (CA) is a potent $\beta$-lactamase inhibitor produced by Streptomyces clavuligerus and has been successfully used in combination with $\beta$-lactam antibiotics (for example, Augmentin) to treat infections caused by $\beta$-lactamase-producing pathogens. Since the discovery of CA in the late 1970s, significant information has accumulated on its biosynthesis, and regarding molecular mechanisms involved in the regulation of its production. Notably, the genes directing CA biosynthesis are clustered along with the genes responsible for the biosynthesis of the $\beta$-lactam antibiotic, cephamycin $\mathrm{C}$, and co-regulated, which makes this organism unique in that the production of an antibiotic and production of a small molecule to protect the antibiotic from its enzymatic degradation are controlled by shared mechanisms. Traditionally, the industrial strain improvement programs have relied significantly on random mutagenesis and selection approach. However, the recent availability of the genome sequence of $S$. clavuligerus along with the capability to build metabolic models, and ability to engineer the organism by directed approaches, has created exciting opportunities to improve strain productivity more efficiently. This review will include focus mainly on the gene organization of the CA biosynthetic genes, regulatory mechanisms that affect its production, and will include perspectives on improving strain productivity.
\end{abstract}

The Journal of Antibiotics (2013) 66, 411-420; doi:10.1038/ja.2013.26; published online 24 April 2013

Keywords: Actinomycetes; $\beta$-lactams; clavulanic acid; secondary metabolism; Streptomyces

\section{INTRODUCTION}

Clavulanic acid (CA) is a potent inhibitor of a wide range of $\beta$-lactamases from pathogenic organisms. ${ }^{1,2}$ It is used in combination with $\beta$-lactam antibiotics against those organisms that are known or suspected to be $\beta$-lactamase producers, to protect activity of the $\beta$-lactam antibiotics and prevent infections. The mechanism of $\beta$-lactamase inhibition is because of the structural similarity of CA with the natural substrate of the $\beta$-lactamase enzyme, that is, $\beta$-lactam antibiotic, which results in CA binding to the serine-hydroxyl group in the active site of the enzyme and causing irreversible enzyme inhibition. ${ }^{3,4} \mathrm{CA}$ is active against group 2 class A or D $\beta$-lactamases, including both penicillinases and cephalosporinases. ${ }^{5}$ The combination therapy of $\mathrm{CA}+\beta$-lactam antibiotic has been very successful in preventing infections due to Gram-positive (Staphylococcus sp.) and Gramnegative (Klebsiella sp. Hemophilus sp. Proteus, Shigella, Pseudomonas.) $\beta$-lactamase-producing pathogens. Augmentin, a combination of CA and the $\beta$-lactam antibiotic, amoxicillin, is a very successful combination therapy with reported global sales of $>\$ 2.1$ billion (Glaxo SmithKline). ${ }^{6,7}$

CA belongs to the clavam group of $\beta$-lactams, characterized by the bicyclic $\beta$-lactam/oxazolidine ring system, in which its $\beta$-lactamase inhibitory property is due to the characteristic $3 \mathrm{R}, 5 \mathrm{R}$ stereochemistry of the strained bicyclic nucleus structure (Figure 1). This $\beta$-lactamase inhibitor was discovered in the fermentation broth of Streptomyces clavuligerus in a screening program aimed at identifying novel $\beta$-lactamase inhibitors. ${ }^{8}$ It is notable that $S$. clavuligerus was previously isolated for its ability to produce the $\beta$-lactam antibiotic, cephamycin $\mathrm{C}$ and related $\beta$-lactam antibiotics (Figure 1), and subsequently it was found that it also produces CA. The $\beta$-lactam antibiotics penicillin $\mathrm{N}$ and cephalosporin occur as intermediates in the production of cephamycin C. Production of both $\beta$-lactam antibiotics and a $\beta$ lactamase inhibitor by one organism, and as described later, the contiguous chromosomal arrangement of the genes responsible for the production of these two classes of compounds, and their coregulation, makes $S$. clavuligerus a unique case, in which both the antibiotic and a molecule to protect the antibiotic from degradation seem to have coevolved. Thus, the idea and development of Augmentin as a combination therapy seems to have been inspired by the mother nature. Subsequently, several other Streptomyces sp., including S. jumonjinensis and $S$. katsurahamanus, have been shown to produce both cephamycin $\mathrm{C}$ and CA. ${ }^{9}$ Among these, S. clavuligerus has been studied the most as a model system, and is also used for producing CA commercially. 
Clavams

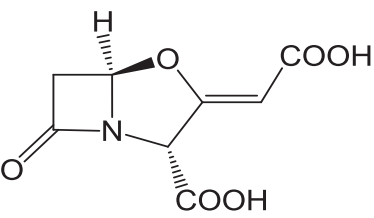

Clavulanic acid

(3R, 5R stereochemistry)

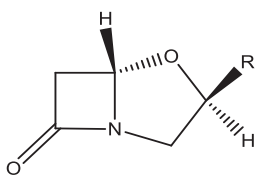

Other clavams

(3S, 5 S stereochemistry)<smiles>CC1(C)S[C@@H]2[C@H](NC(=O)CCC[C@H](N)C(=O)O)C(=O)N2[C@H]1C(=O)O</smiles><smiles>N[C@H](CCCCC(=O)N[C@@H]1C(=O)N2C(C(=O)O)=C(CO)CS[C@H]12)C(=O)O</smiles><smiles>CO[C@@]1(NC(=O)CCCC[NH3+])C(=O)N2C(C(=O)O)=C(CC(=O)O)CS[C@H]21</smiles>

Figure $1 \beta$-Lactam metabolites produced by $S$. clavuligerus.

Apart from cephamycin C and CA, S. clavuligerus also produces non-CA clavam type of compounds all of which contain the antipodal $3 \mathrm{~S}$, 5S stereochemistry in their $\beta$-lactam ring system, as compared with the $3 \mathrm{R}, 5 \mathrm{R}$ stereochemistry of $\mathrm{CA}$, which is required for the $\beta$-lactamase inhibitory property ${ }^{10}$ (Figure 1 ). Therefore, the $3 \mathrm{~S}$, 5S clavams do not possess any $\beta$-lactamase inhibitory property, but do possess activities ranging from antibacterial and antifungal in nature. Owing to structural similarity between CA and 3S, 5S clavams, there are shared mechanisms involved in their biosynthesis. ${ }^{11,12}$ However, this review will focus primarily on the molecular aspects of CA biogenesis in S. clavuligerus, with special emphasis on the strategies used for strain development. The biosynthesis of 3S, $5 \mathrm{~S}$ clavams and relevant genetics have been reviewed elsewhere. ${ }^{13,14}$

\section{BIOGENESIS}

The knowledge on CA biosynthesis has been obtained from several lines of investigation including classical approaches of feeding cells with labeled precursors and tracing the incorporation of the label in the CA molecule, ${ }^{10,15-17}$ isolating or constructing non-producing mutants ${ }^{13,18,19}$ and characterizing the accumulated intermediates, and taking molecular approaches including, sequence and transcriptional analysis of the biosynthetic and regulatory genes. ${ }^{13}$ The biosynthetic pathway for CA is presented in Figure 2, and the description of enzymes and genes involved in the pathway and their functions is summarized in Table 1. The CA biosynthetic gene cluster is located immediately downstream from the cephamycin C gene cluster, ${ }^{20,21}$ and stretches from carboxyethylarginine synthase ceaS2 to gcaS; $;^{13}$ (Figure 3). Beyond ceaS2 on one end of the CA cluster is $p c b R$, a penicillin-binding protein involved in $\beta$-lactam resistance and part of the cephamycin $\mathrm{C}$ gene cluster. ${ }^{22}$ Beyond gcaS on the other end are orf-18 and orf-19, encoding another set of penicillin-binding proteins, which are not involved in CA biosynthesis. These are followed by orf-20, orf-21 and orf-22, encoding potential regulatory genes, which seem to have some effects on CA production. However, these genes also show pleiotropic effects, so their role in CA biosynthesis remains uncertain $^{13,23,24}$ (Figure 3).

\section{THE 'EARLY' STEPS}

The CA pathway begins with the condensation of the 3-carbon glyceraldehyde 3-phosphate with the 5-carbon L-arginine to form N2-(2-carboxyethyl) arginine, catalyzed by carboxyethylarginine synthase (CeaS2). ${ }^{25}$ In the next reaction, N2-(2-carboxyethyl) arginine is cyclized to form a monocyclic $\beta$-lactam compound, deoxyguanidinoproclavaminate, catalyzed by $\beta$-lactam synthase (Bls2). ${ }^{26-28}$ Deoxyguanidinoproclavaminate is then hydroxylated to form guanidinoproclavaminate, catalyzed by clavaminate synthase (Cas2). ${ }^{29-31}$ Guanidinoproclavaminic acid undergoes a hydrolysis reaction catalyzed by proclavaminate amidino-hydrolase (Pah2) to form proclavaminate. ${ }^{21,32}$ The next two consecutive reactions are again catalyzed by Cas2. In the first reaction, proclavaminate is cyclized to form the first bicyclic ring structure, dihydroclavaminic acid, which then undergoes subsequent desaturation to produce clavaminic acid. ${ }^{33}$ The enzymology involved in the 'early' reactions leading to the formation of clavaminic acid is well characterized and reviewed elsewhere. ${ }^{13}$ The genes encoding the 'early' steps, ceasS2, bls, pah and cas2, are coexpressed and coregulated. The gene immediately downstream from cas-2 is oat2, which codes for an ornithine acetyltransferase-like activity. ${ }^{18,34}$ On the basis of mutational analysis and similarity of its transcriptional regulation to that of 'early' genes, it is considered a part of the 'early' gene cluster. However, oat2 is transcribed separately. ${ }^{34,35}$ The oat2 mutant showed decreased CA production, however, this phenotype could be reversed by addition of arginine. Therefore, Oat2 is thought to contribute toward building arginine pools required for CA biosynthesis.

It is striking to note that in S. clavuligerus the entire 'early' gene cluster is duplicated. This knowledge came from several independent studies. The initial enzymology and gene cloning had demonstrated the existence of duplicate sets (paralogs) of Cas genes, cas1 and cas2, located on two different regions of the chromosome. ${ }^{30}$ cas 2 is now assigned to the CA gene cluster downstream from the cephamycin $\mathrm{C}$ gene cluster along with other 'early' CA biosynthetic genes, whereas cas 1 resides elsewhere on the chromosome along with 3S 5S clavamspecific genes. ${ }^{36,37}$ Separately, it was discovered that knock-out 


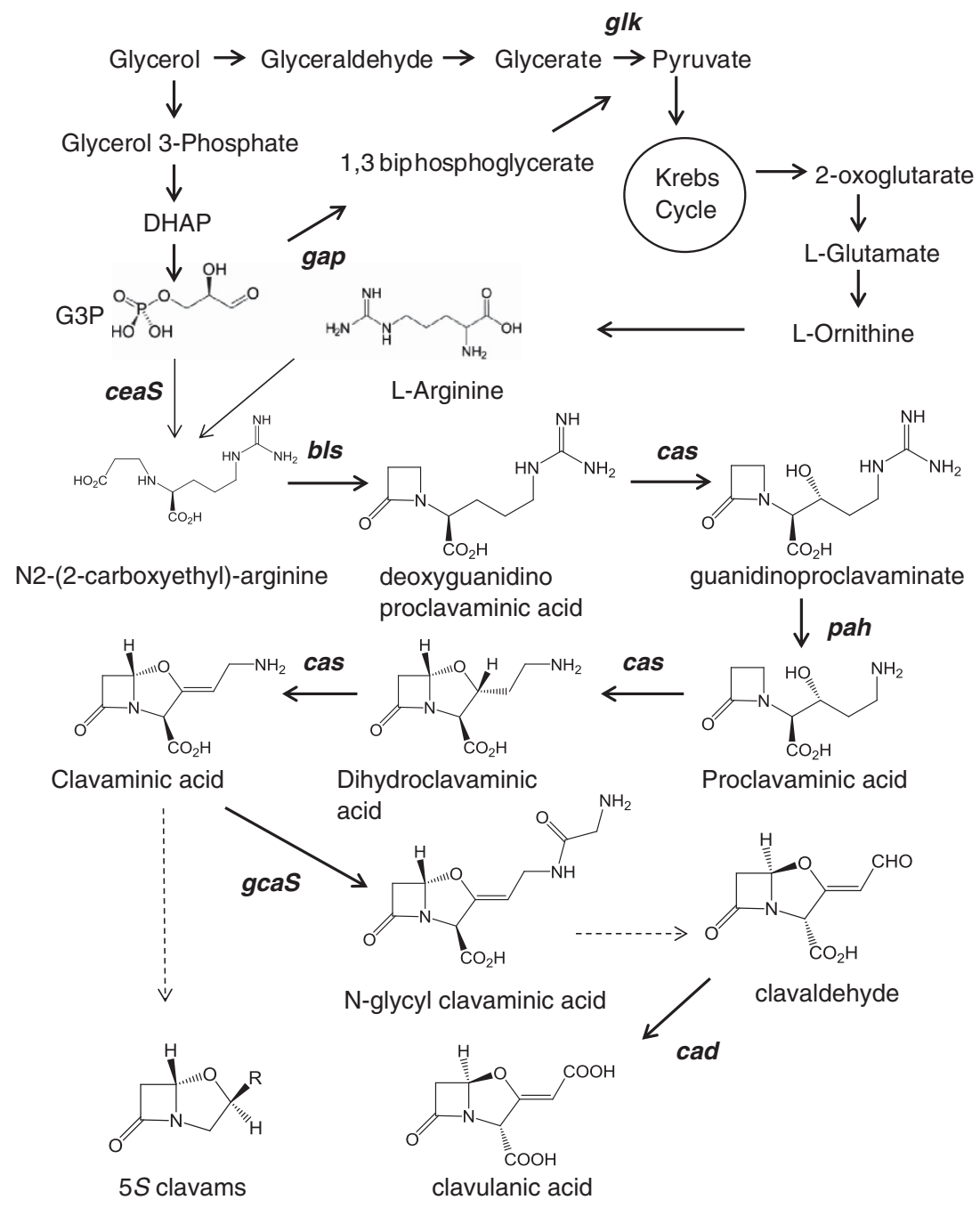

Figure 2 Clavulanic acid (CA) biosynthetic pathway. gap, glyceraldehyde-3-phosphate dehydrogenase; glk, glycerate kinase.

mutations in each of the five 'early' CA biosynthetic genes, but not in the 'late' genes, led to a 'leaky' phenotype; that is, the mutants in each of the five genes, ceasS2, bls2, pah2, cas2 and oat2, were completely blocked in CA production when cultures were grown in a synthetic medium. However, the same mutants were still able to produce CA in a complex soy-based medium. ${ }^{18}$ This suggested the presence of a second set of the 'early' genes, which was expressed in the soy-based medium but not in the synthetic medium, and which functionally complemented the mutations in the 'early' genes, in the soy-based medium. Supporting this idea, transcriptional analysis confirmed that cas 2 was expressed in both the synthetic medium and the soy-based medium, whereas its paralog, casl, was expressed in the soy medium, but not the synthetic medium. ${ }^{18,38}$ Eventually, the second set of the paralogous 'early' genes was located, characterized, and their role in the biosynthesis of 3S, $5 \mathrm{~S}$ clavams established. Of these, ceasS1, bls1, pah1 and oat 1 are clustered with 3S-, 5S-specific clavam genes (the paralog gene cluster), and located on the giant linear plasmid pSCL4, whereas cas 1 is colocated with yet another set of clavam biosynthetic genes (clavam biosynthetic gene cluster) and is present on the chromosome, well separated from the cephamycin C-CA gene cluster by $>1 \mathrm{Mb}^{36,37,39-41}$ The gene organization of the paralog gene cluster and the clavam biosynthetic gene cluster responsible for the production of $3 \mathrm{~S}, 5 \mathrm{~S}$ clavams has been recently reviewed elsewhere. ${ }^{13}$

In summary, this organism has two sets of the 'early' genes leading to the formation of clavaminic acid, in three locations on the chromosome. One complete set is clustered with the 'late' genes responsible for CA biosynthesis; that is, the cephamycin C-CA gene cluster, whereas the second set of genes is located within two separate clusters responsible for the $3 \mathrm{~S}$, $5 \mathrm{~S}$ clavam biosynthesis.

\section{THE 'LATE' STEPS}

Clavaminic acid arising from the 'early' pathway is the branch point intermediate that can either be converted to $\mathrm{CA}$, or to the $3 \mathrm{~S}$, $5 \mathrm{~S}$ clavams, by respective 'late' reactions. The 'late' steps involved in the formation of 3S, $5 \mathrm{~S}$ clavams have been discussed elsewhere. ${ }^{13}$

The 'late' steps involved in the conversion of clavaminic acid to CA proceed via clavaldehyde as an intermediate, and entail two key structural modifications. The first modification is the inversion of the stereochemistry from $3 \mathrm{~S}, 5 \mathrm{~S}$ in clavaminic acid to $3 \mathrm{R}, 5 \mathrm{R}$ in clavaldehyde and $\mathrm{CA}$; that is, a double ring enantiomerization reaction, which gives these later compounds their characteristic $\beta$-lactamase-inhibitory property. The second modification is the conversion of the amino group present on the side chain of 
Table 1 Genes involved in clavulanic acid (CA) pathway and their role and function

\begin{tabular}{|c|c|c|c|c|}
\hline Gene/ORF & Protein/ldentity & Function/Putative role & Mutant phenotype & Reference \\
\hline \multicolumn{5}{|c|}{ Common early pathway (conversion of early precursors to clavaminic acid) } \\
\hline ceas2 (pyc), ceas1 & $\begin{array}{l}\text { Carboxyethylarginine } \\
\text { synthase }\end{array}$ & $\begin{array}{l}\text { Condensation of L-arginine and glyceraldehyde- } \\
\text { 3-phosphate to produce N2-(2-carboxy-ethyl) } \\
\text { arginine. }\end{array}$ & & 25 \\
\hline bls2, bls1 & $\beta$-lactam synthase & $\begin{array}{l}\text { Cyclization of N2-(2-carboxy-ethyl) arginine to } \\
\text { form deoxyguanidinoproclavaminate }\end{array}$ & & $26-28$ \\
\hline cas2, cas 1 & Clavaminate synthase & $\begin{array}{l}\text { 1.Hydroxylation of deoxyguanidino-proclavami- } \\
\text { nate to form guanidine-proclavaminate. } 2 \text {. } \\
\text { Cyclization of proclavaminate to form } \\
\text { dihydroclavaminate.3. Desaturation of } \\
\text { dihydroclavaminate to form clavaminate. }\end{array}$ & $\begin{array}{l}\text { Conditional loss of CA production owing to } \\
\text { the presence of a second set of paralogous } \\
\text { genes, ceaS1, b/s } 1 \text {, cas } 1 \text {, pah } 1 \text { and oat } 1 \text {, } \\
\text { present in the chromosome clustered with } \\
\text { genes specific for } 5 \mathrm{~S} \text { clavam production. }\end{array}$ & $29-31,33$ \\
\hline pah2, pah1 & $\begin{array}{l}\text { Proclavaminate } \\
\text { amidinohydrolase }\end{array}$ & $\begin{array}{l}\text { Hydrolysis of guanidine-proclavaminate to form } \\
\text { proclavaminate. }\end{array}$ & & 21,32 \\
\hline oat2, oat1 & $\begin{array}{l}\text { Ornithine } \\
\text { acetyltransferase }\end{array}$ & $\begin{array}{l}\text { Transfer of an acetyl group from } \mathrm{N} \text {-acetylor- } \\
\text { nithine to glutamate; increase cellular pools of } \\
\text { arginine via glutamate. }\end{array}$ & & $18,34,35$ \\
\hline \multicolumn{5}{|c|}{ Late pathway (conversion of clavaminic acid to CA) } \\
\hline OppA1 (orf-7) & $\begin{array}{l}\text { Oligopeptide permease- } \\
\text { binding proteins }\end{array}$ & Unknown & Loss of CA production & $18,27,46,47$ \\
\hline claR (orf-8) & $\begin{array}{l}\text { LysR type transcrip- } \\
\text { tional activator }\end{array}$ & Positively regulates 'late' steps of the pathway & $\begin{array}{l}\text { Loss of CA production } \\
\text { Accumulation of clavaminic acid } \\
\text { Loss of oppA1 (orf- } 7 \text { ), cad (orf-9) and cyp } \\
\text { (orf-10) transcripts }\end{array}$ & 44,48 \\
\hline cad (orf-9) & $\begin{array}{l}\text { Clavaldehyde } \\
\text { dehydrogenase }\end{array}$ & Reduction of clavaldehyde to CA & Loss of CA production & $18,44,45$ \\
\hline $\operatorname{cyp}($ orf-10) & Cytochrome P450, & $\begin{array}{l}\text { Oxidative reactions; role in ring double inversion } \\
(3 \mathrm{~S}, 5 \mathrm{~S} \text { to } 3 \mathrm{R}, 5 \mathrm{R}) \text { and clavaldehyde formation? }\end{array}$ & Loss of CA production & $18,46,49$ \\
\hline$f d(o r f-11)$ & Ferredoxin & Electron transfer & Partial loss of CA production & $18,46,49$ \\
\hline Orf-12 & $\begin{array}{l}\text { Similarity to class } A \\
\beta \text {-lactamases }\end{array}$ & $\begin{array}{l}\text { Ring opening/closure of } \beta \text {-lactam ring during } \\
\text { ring inversion? }\end{array}$ & Loss of CA production & 43,49 \\
\hline Orf-13 & Amino acid efflux pump & Transport of clavam metabolites outside the cell & $\begin{array}{l}\text { Severe loss of CA production and } 5 \text { S clavam } \\
\text { production }\end{array}$ & 43,46 \\
\hline Orf-14 & $\begin{array}{l}\text { Gcn5-related acetyl } \\
\text { transferases }\end{array}$ & $\begin{array}{l}\text { Role in biosynthesis/resistance? } \\
\text { Acetylation of } \mathrm{N} \text {-glycylclavaminic acid to form } \\
\mathrm{N} \text {-acetylglycylclavaminic acid? }\end{array}$ & $\begin{array}{l}\text { Loss of CA production } \\
\text { Accumulation of NG-clavaminic acid } \\
\text { Partial loss of CA }\end{array}$ & $43,46,50$ \\
\hline OppA2 (orf-15) & $\begin{array}{l}\text { Oligopeptide permease- } \\
\text { binding proteins }\end{array}$ & Unknown & $\begin{array}{l}\text { Loss of CA production } \\
\text { Accumulation of NAG-clavaminic acid and } \\
N \text {-glycylclavaminic acid }\end{array}$ & $18,27,46,47$ \\
\hline Orf-16 & $\begin{array}{l}\text { No significant homology } \\
\text { to proteins of known } \\
\text { function }\end{array}$ & $\begin{array}{l}\text { Role in conversion of } \mathrm{N} \text {-acetylglycylclavaminic } \\
\text { acid to } \mathrm{N} \text {-acetylclavaminic acid? }\end{array}$ & $\begin{array}{l}\text { Loss of CA production } \\
\text { Accumulation of NAG-clavaminic acid } \\
\text { Accumulation of traces of NG-clavaminic } \\
\text { acid }\end{array}$ & 43,46 \\
\hline gcas (orf-17) & $\begin{array}{l}\text { Glycylclavaminate } \\
\text { synthase }\end{array}$ & Clavaminate to $\mathrm{N}$-glycylclavaminate & Loss of CA production & 42,43 \\
\hline
\end{tabular}

clavaminic acid to a hydroxyl group, via an oxidative deamination reaction. However, reactions involved in the two modifications are not clearly understood. The DNA sequencing of the putative 'late' genes and characterization of the corresponding knock-out mutants have provided some insights into the nature of potential 'late' steps of the pathway.

The first dedicated 'late' reaction is the conversion of clavaminic acid to $N$-glycylclavaminic acid, catalyzed by glycylclavaminic acid synthase (gcaS; orf-17). ${ }^{42,43}$ It is predicted that the double ring enantiomerization and oxidative deamination reactions then follow, leading to the formation of clavaldehyde, which possesses the $3 R, 5 R$ stereochemistry essential for the $\beta$-lactamase activity. However, the details of these reactions and how $N$-glycylclavaminic acid is converted to clavaldehyde remain elusive. The last reaction entails reduction of clavaldehyde to $\mathrm{CA}$, catalyzed by NADPH-dependent clavaldehyde dehydrogenase ( $\mathrm{cad}) .{ }^{18,44,45}$

Aside from $\mathrm{gcaS}$ and $\mathrm{cad}$, there are at least six additional genes that seem to be essential for CA biosynthesis, as mutations in these genes lead to loss of CA production. The exact role of these genes in the pathway is not fully understood, although sequence similarities and 


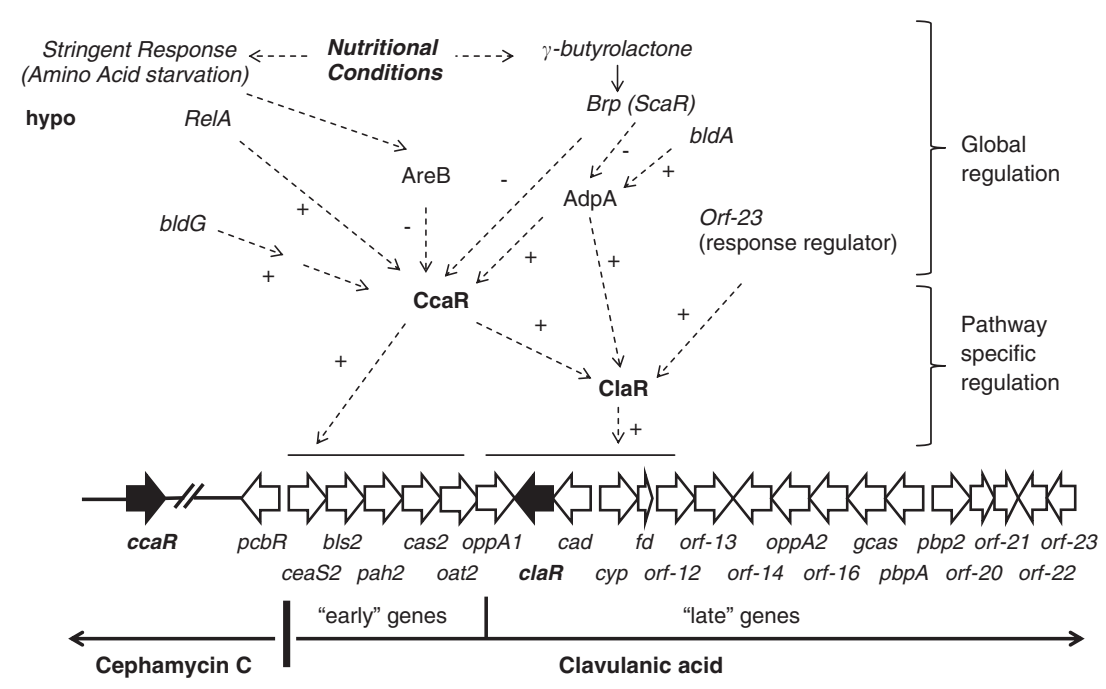

Figure 3 Clavulanic acid (CA) biosynthetic gene cluster and its regulation (a hypothetical scheme based on published work and references cited in this article).

analysis of knock-out mutants have provided some clues into their function.

oppA1 (orf-7) and oppA2 (orf-15) encode similar proteins with sequence similarity to periplasmic oligopeptide-binding proteins. ${ }^{18,27,46,47}$ Mutants in each of these genes are unable to produce CA, suggesting that these genes are essential for CA production, but also that these genes cannot complement each other. ${ }^{47}$ However, the exact role of the putative peptide transport function in CA production is unclear. Perhaps these two gene products are responsible for transporting arginine/peptide substrates or regulatory signaling peptides involved in CA biosynthesis. ${ }^{47}$

claR (orf-8) encodes a transcriptional activator-containing sequences with significant similarity to the helix-turn-helix motif of the LysR family of transcriptional regulators, and positively regulates the 'late' genes oppA1, cad and cyp. ${ }^{44,48}$ A claR mutant was blocked for CA production but accumulated clavaminic acid. In the same mutant, transcripts for 'early genes' ceas $S 1$, bls1, pah1, cas2 and oat 1 remained unaffected, whereas the transcripts corresponding to the 'late' genes oppA1, cad and cyp were absent. ${ }^{44,48}$ These data are consistent with the role of claR as a regulator of 'late' steps specific to the conversion of clavaminic acid to CA.

cyp (orf-10) encodes cytochrome P450 (Cyp) hydroxylase whereas $f d$ (orf-11) encodes a ferredoxin, an electron transport protein, which could provide functionality to the Cyp protein. ${ }^{18,46,49}$ A mutation in cyp leads to loss of CA formation, whereas a mutation in $f d$ results in partial loss, suggesting that another electron transfer protein from the organism may be able to partner with Cyp in the absence of the Fd. The $c y p-f d$ combination of genes is a potential candidate for carrying out the oxidative deamination and/or double ring enantiomerization reactions present between clavaminic acid and CA, although the in vitro evidence is yet to be established. ${ }^{18,46,49}$

orf-12, which has some similarity to class A $\beta$-lactamases, is essential for CA production based on mutational analysis. ${ }^{42,49}$ Orf- 12 encodes a protein with some similarity to $\beta$-lactamases and possesses the highly conserved SDN motif important for the catalytic activity of $\beta$-lactamases. However, it lacks the other conserved motifs, STFK, EPELN and KTG. The role of orf- 12 in CA biosynthesis is not clear as yet.

orf-13 encodes an amino acid metabolite efflux pump protein and may be involved in the transport of CA and pathway intermediates from inside of the cell to the outside. ${ }^{42,46}$ Efflux pumps are commonly associated with antibiotic gene clusters as a part of the resistance mechanism to protect the organism from excess build-up of potentially toxic metabolites. The mutation in orf-13 decreases CA production significantly. ${ }^{42,46}$

orf-14 shows similarities to GCN5-acetyltransferases, and mutants in this gene are negatively affected in CA production. The acetylation could have a role in the generation of $\mathrm{N}$-acetylated clavaminic acid intermediates, which have been detected earlier in uncharacterized mutants of $S$. clavuligerus. ${ }^{42,46,50}$

orf-16 shows no significant similarities to any proteins in the database. However, a mutation in orf-16 leads to a complete loss of CA formation, demonstrating the essential nature of this gene for CA production. ${ }^{42,46}$ Interestingly, orf-16 showed accumulation of $\mathrm{N}$-acetylglycylclavaminic acid and $\mathrm{N}$-glycylclavaminic acid metabolites. The accumulation of the modified clavaminic acid metabolites in orf-16 mutants, and the putative acetylation role of orf-14, suggests that clavaminic acid is first converted to glycylclavaminic acid catalyzed by gcaS, then acetylated by orf-14 gene product to form $\mathrm{N}$-acetylglycylclavaminic acid, and subsequently converted to $\mathrm{N}$-acetylclavaminic acid by orf-16 gene product. However, more experimental data need to be generated to confirm this hypothesis.

\section{REGULATION}

Most antibiotic biosynthetic gene clusters in Streptomyces are regulated in a hierarchical manner. ${ }^{51}$ At the first level, the biosynthetic genes are typically controlled by pathway-specific transcriptional regulators encoded within the gene clusters. The expression of the transcriptional regulators in turn is tightly controlled by global regulatory mechanisms, which tie the production of the antibiotic to the physiological state of the cell and morphological differentiation (formation of aerial mycelium and sporulation). The evidence suggests that CA biosynthesis in S. clavuligerus is also regulated in this hierarchical manner ${ }^{52}$ (Figure 3).

At the bottom of the hierarchy is claR, which, as discussed earlier, is a LysR type of transcriptional activator located within the CA gene cluster and positively regulates only the 'late' biosynthetic genes involved in the conversion of clavaminic acid to CA (see above).

At the next level is $c c a R$, which is located within the adjacent cephamycin C gene cluster, and encodes a Streptomyces antibiotic 
regulatory protein family, which shows similarity to the OmpR family of regulators but lacks any evidence of a helix-turn-helix DNA-binding motif. ${ }^{53-55}$ Knock-out mutation of $c c a R$ abolishes both cephamycin $\mathrm{C}$ and CA production, which is restored by introducing the wild-type $c c a R$ back into the mutants, suggesting that CcaR positively regulates both cephamycin $\mathrm{C}$ and $\mathrm{CA}$ production. Transcriptional analysis suggests that $\mathrm{CcaR}$ regulates $\mathrm{CA}$ production directly, by regulating the expression of the 'early' ceaS2-bls-pah-cas2 polycistronic transcript, and indirectly, by regulating expression of claR, which in turn controls expression of the 'late' genes in the CA pathway. Heptameric target sequences upstream of ceaS2 and claR, where $C c a R$ is thought to bind and exert transcriptional control, have been identified via gel-shift and DNA footprinting analysis. ${ }^{55}$ These sequences are similar to target sequences where the Streptomyces antibiotic regulatory protein family of transcriptional regulators binds and regulates gene expression in other Streptomyces. CcaR also binds to the sequences upstream from $c c a R$ and autoregulates its own expression, indicating the presence of an added level of control to fine tune the production of the two $\beta$-lactams in this organism. ${ }^{53-55}$

The next level of hierarchy involves several levels of global regulatory mechanisms, which allows the organism to trigger the onset of antibiotic production and morphological differentiation in response to different environmental and metabolic signals. ${ }^{51}$ Such signals include changes in growth rate, nutritional stress and environmental imbalances. These mechanisms can operate at the level of transcription, translation or post-translation of key effector genes/gene products responsible for further cascading the signal to regulate the expression of the target genes. In some cases, small MW compounds, such as $\gamma$-butyrolactones, are known to mediate such effects. Although the data from several laboratories suggests that the CA gene cluster is regulated by such global mechanisms, there are some notable differences as compared with similar mechanisms operating in other Streptomycetes. The following section covers three global regulatory mechanisms, which seem to be operating in S. clavuligerus, including those involving (a) bld genes, (b) production of autoregulators and (c) stringent response.

In Streptomyces, the bld gene-based regulon links the expression of antibiotic gene clusters to morphological differentiation, and common genes and mechanisms have been identified that regulate both antibiotic production and morphological differentiation. One such gene is bldA that encodes a leucyl-transfer RNA that recognizes UUA codons, and is involved in the translation of genes containing TTA codons. The TTA-encoding genes in Streptomyces are rare and present only in secondary metabolism regulatory genes, or genes associated with the regulation of sporulation and aerial mycelium formation. ccaR, which controls both cephamycin C and CA gene clusters, contains the rare TTA codon, which initially suggested the presence of this mode of regulation. ${ }^{54,56}$ However, although bldA mutants of $S$. clavuligerus show the absence of aerial mycelium formation, as expected of this phenotype, the same mutants are not blocked for production of either CA or cephamycin C. This suggests that there must be an alternative mechanism for translation of the ccaR TTA codons, or mistranslation mechanism in the absence of bldA transfer RNA, and also suggests that CA and cephamycin C gene clusters are independent of the bldA-mediated regulation, at least under the conditions tested. ${ }^{56}$ This is an exception to the bldAmediated control of the TTA-encoding target genes normally seen in other Streptomyces systems.

Another mode of regulation that commonly controls antibiotic production in Streptomyces involve sigma factors and sigma factorrelated mechanisms, like those involving anti-sigma factors and anti-anti-sigma factors. $^{51}$ Transcriptional regulation by sigma factor-related mechanisms is common in Bacillus sp. for controlling sporulation and other stress-associated responses. The presence of more than 60 sigma factors present in the S. coelicolor genome suggests that sigma factors-based regulation is also present in this organism. ${ }^{57}$

bld $G$ encodes a putative anti-anti-sigma factor, which is thought to regulate those genes involved in morphological differentiation that are regulated by sigma factors. In $S$. clavuligerus, bldG mutants were blocked in morphological differentiation and production of both CA and cephamycin $\mathrm{C}$, indicating the presence of bldG-mediated regulation in this organism. ${ }^{58,59}$ Transcriptional analysis revealed that in the bldG mutant, $c c a R$ transcription was abolished. Thus, it appears that the bldG in S. clavuligerus controls CA and cephamycin C production by regulating transcription of $c c a R$, perhaps mediated by other as yet unidentified proteins in the regulatory cascade. ${ }^{58,59}$ Similarly, orf-21 located downstream of $\mathrm{gcaS}$ within the CA gene cluster codes for a putative sigma factor, which appears to have a role in CA production. However, its effect on CA production was variable across different laboratories, and seems to be linked closely with growth conditions. ${ }^{13,24,60}$

The two-component sensor kinase-response regulator system is another mechanism present in Streptomyces to regulate antibiotic production and morphological differentiation in response to the physiology of the organism. There are more than 80 sensor kinases and more than 60 response regulators present in the $S$. coelicolor genome. ${ }^{57}$ The two genes downstream from the CA gene cluster, orf-22 and orf-23, show similarity to sensor kinases and response regulators, respectively, from other Streptomyces. ${ }^{24,61}$ Although the orf- 22 mutation had little effect on CA production, orf-23 mutant showed severe reduction in CA levels, decreased levels of claR transcripts, and defects in growth and sporulation. Conversely, overexpression of orf- 23 caused overproduction of spores and CA. This phenotype suggests that orf-23 may act as a response regulator to control growth, sporulation and antibiotic production in this organism in response to environmental signals.

The $\gamma$-butyrolactone (autoregulatory)-mediated regulation of morphological differentiation and antibiotic production is prevalent in Streptomyces. ${ }^{51}$ In this model of regulation, the autoregulators bind to their cognate-binding proteins, called as autoregulator-binding proteins, which in turn, bind to regulatory sequences (ARE) upstream of the target genes; that is, genes directly or indirectly involved in differentiation and antibiotic production to regulate gene expression at the transcriptional level. ${ }^{62,63}$ The evidence so far suggests that the autoregulator-binding protein ARE-mediated regulation is also present in S. clavuligerus, and controls CA and cephamycin C production via $c c a R^{64,65}$ Although small molecule autoregulators have been identified in this organism, ${ }^{66}$ the exact small molecule involved in this particular interaction has not yet been identified. ScaR (also called the Brp; $\gamma$-butyrolactone receptor protein), an autoregulator-binding protein, was identified in this organism, ${ }^{64}$ along with the corresponding ARE target sequences upstream from $c c a R{ }^{64,67}$ The binding of ScaR to $c c a R$ ARE sequences has been demonstrated in vitro, and scaR mutants overproduce both CA and cephamycin $\mathrm{C}$ significantly. This suggests that ScaR protein interacts with its cognate small molecule (as yet unidentified), and then binds to the $c c a R$ ARE sequences, and represses the $c c a R$ transcription.

Although Brp ( $\mathrm{ScaR}$ ) is postulated to control $c c a R$ directly, it also controls $c c a R$ and $c l a R$ expression indirectly, via the AdpA pathway. ${ }^{68}$ AdpA is a transcriptional regulator that shows similarity to the AraC/XylS family of transcriptional activators, contains two helix- 
turn-helix motifs and a UUA leucine codon in the coding region. AdpA has been characterized in S. griseus and S. coelicolor where it regulates sporulation, morphogenesis and antibiotic production. In S. clavuligerus, adpA expression is regulated negatively by ScaR (Brp), and thus, in brp-mutants, adpA expression is elevated. The adpA mutants of $S$. clavuligerus show a significant decrease in CA production, and also decreased transcripts levels of $c c a R, c l a R$, the 'early' and other biosynthetic genes. These data suggest that Brp negatively regulates AdpA, which, in turn, positively regulates ccaR and thus CA production. Furthermore, as adpA contains a UUA leucine codon and is not expressed in bldA mutant of S. clavuligerus, adpA provides linkage between bldA regulation and CA production.

Apart from ScaR, AreB is another protein that has been shown to bind to $c c a R$ ARE sequences in gel-shift DNA-binding experiments. ${ }^{69}$ AreB belongs to the IclR family of regulatory proteins, and the corresponding gene areB is located adjacent to the leuCD operon, in opposite transcriptional orientation. The areB autoregulates its own transcription and also regulates transcription of leuCD operon. The areB mutant displays weak leucine auxotrophy, and a derepression effect on CA production, suggesting this mechanism could provide a link between primary and secondary metabolism. ${ }^{69}$ However, the derepression effect of the areB mutation on CA production and $c c a R$ transcript levels was modest as compared with similar effects due to $s c a R$ mutation. Interestingly, the binding of AreB to $c c a R$ ARE sequences requires a small molecule, as purified protein does not show the binding activity. The identity of this small molecule autoregulator remains to be established.

The stringent response, which is a well-studied phenomenon in E. coli, is also involved in regulating secondary metabolism in Streptomyces including CA biosynthesis in S. clavuligerus. This mechanism involves production of unusual phosphorylated nucleotide (p)ppGpp, mediated by two proteins, ppGpp synthetase (RelA) and (p)ppGpp synthetase/hydrolase (SpoT). ${ }^{51}$ These phosphorylated nucleotides have a regulatory role via binding to the $\beta$-subunit of RNAP, and regulating expression of ribosomal genes, and resulting protein synthesis, in response to amino-acid starvation and nutritional downshift conditions. In S. coelicolor, the corresponding RelA/SpoT homologs, RelA/RshA, have been identified and shown to control antibiotic biosynthesis, at least in certain physiological conditions. ${ }^{51}$ Similarly, in S. clavuligerus, a relA mutant was significantly blocked in its ability to produce both CA and cephamycin C, and the mutant was also impaired in aerial mycelium formation and sporulation; however, a less drastic effect was observed for $r s h A$ mutants. ${ }^{70,71}$ On the basis of this evidence, it is likely that in S. clavuligerus, the stringent response to amino-acid starvation induces production of the phosphorylated nucleotides, mediated by RelA/RshA, and these nucleotides could positively influence CA production by mechanisms that need to be further elucidated.

\section{STRAIN DEVELOPMENT}

The strain development strategies to deliver microbial products at higher yields and lower costs have evolved from the traditional random mutation and selection approach to knowledge-based rational approaches. The latter relies upon understanding key biosynthetic and regulatory mechanisms affecting the yields, and engineering strains to eliminate the potential bottlenecks in a targeted manner. There are successful examples of the application of both these approaches for improving CA-producing S. clavuligerus strains.

Owing to the proprietary nature of the industrial screening programs, not much published literature is available, discussing specific screening strategies employed for developing high
CA-producing strains. However, given that the titers of wild-type ATCC strain are reportedly in the range of 25-120 $\mathrm{mgl}^{-1}$ (depending on the media and growth conditions, which varies from laboratory to laboratory), and a recent report of titers in an industrial strain approaching $3 \mathrm{gl}^{-1}, 6,72$ it suggests that the industrial strain improvement programs have enabled orders of magnitude improvement in CA strain productivity.

More recently, the knowledge gained on the biogenesis of CA and the availability of genetic tools in S. clavuligerus to overexpress genes, and/or to eliminate competing reactions by making directed modifications has allowed construction of improved strains in a rational manner. These efforts can be broadly classified into three approaches: (a) increasing the flow of precursors into the pathway, (b) increasing the gene dosage of key biosynthetic and/or regulatory genes and (c) eliminating competing reactions that divert the flow of pathway intermediates into non-CA products, or competing reactions that affect the overall energetics and carbon flow into the CA pathway.

As discussed earlier, arginine and glyceraldehyde-3-phosphate (G3P) are the two essential precursors of the CA pathway (Figure 2). Earlier reports that supplementation of glycerol leads to an increase in CA yields, suggested that G3P could be a limiting factor. Once glycerol is metabolized into G3P, it can either enter the glycolytic reactions and then the Krebs cycle or it can be recruited into either gluconeogenesis to produce glucose, or it can combine with arginine to begin CA biosynthesis. Thus, a gene encoding glyceraldehyde-3-phosphate dehydrogenase ( $g a p$ ), which is involved in channeling G3P into the glycolytic pathway via formation of 1,3 , diphosphoglyceric acid and pyruvate (Figure 3), was identified, and the disrupted gap-1 mutant showed substantial increase (80-110\%) in CA yields over the wild type, suggesting that in the gap-1 mutant more G3P pools were now available for CA biosynthesis. ${ }^{73}$ Further increases of $>200 \%$ were obtained when arginine was fed to the gap-1 mutants, which suggests that when increased pool of G3P was available, arginine became limiting for CA biosynthesis. ${ }^{73}$ Efficient glycerol utilization to provide sufficient G3P pools also determines the CA yields. Increased gene dosage of $g l p$ operon consisting of glycerol transporter, glycerol kinase, and glycerol-3-phosphate dehydrogenase increased the CA yields further by $>4.5$-fold, and $\sim 7.5$-fold upon supplementation with glycerol. ${ }^{74}$

Increasing gene dosage of key CA biosynthetic and/or regulatory genes has allowed construction of improved strains in a rational manner resulting in productivity increases in the range anywhere between 2- and $>20$-fold. ${ }^{23}$ Introduction of additional gene copies by using integrative vectors or by use of multi-copy plasmids has given promising results. The integration approach seems more practical; considering it provides higher strain stability and obviates the use of antibiotics for plasmid selections. Increasing gene dosage of the 'early' pathway genes, ceaS2, blsS2, cas2 and pah2, either individually or as an operon, and 'late' pathway genes cyp, $f d$, orf-12 and orf-14, resulted in increased production levels. ${ }^{23,75-77}$ Similarly, increasing gene dosage of the two pathway-specific regulators $c c a R$ and $c l a R$ resulted in increased strain productivity. In one case, increasing gene dosage of cas 2 along with $c c a R$ reportedly gave more than a 20 -fold increase in CA titer. In another strategy, $c c a R$ and $c l a R$ were overexpressed in a gap-1 mutant, giving significant increase in titers, which were further enhanced upon feeding the strain with glycerol and ornithine, presumably leading to higher levels of the two precursors, G3P and arginine, being available for CA biosynthesis. ${ }^{78}$ Thus, combining appropriate mutations with increased dosage of genes determining rate-limiting reactions in a logical manner, coupled with appropriate feeding strategies is critical for enhancing CA titers. 
Elimination of competing pathways, which draw upon common pathway intermediates, or influence the overall energetics and primary metabolic pools available for CA biosynthesis, is another strategy that has been successfully applied to construct high-yielding strains. ${ }^{79}$ When $c v m 1$, a gene encoding an enzyme specifically involved in the conversion of the branch point intermediate clavaminic acid to $3 \mathrm{~S}$, $5 \mathrm{~S}$ clavams, was inactivated it resulted in increased CA production, presumably resulting from increased channeling of clavaminic acid to $\mathrm{CA}^{79}$ Interestingly, although the cephamycin $\mathrm{C}$ pathway draws upon $\alpha$-aminoadipate, L-cysteine and L-valine as precursors and is biochemically distinct from CA pathway, its elimination from the strain resulted in increased CA titers. Disruption of lat gene, encoding lysine $\varepsilon$-aminotransferase that converts lysine to $\alpha$-aminoadipate, resulted in loss of cephamycin $\mathrm{C}$ production as expected, but also resulted in approximately $200-250 \%$ increase in CA production as compared with the wild type. ${ }^{79}$ Both cvm1 and lat mutations were subsequently introduced into a high titer commercial strain, which resulted in a consistent $10 \%$ increase in CA titers over the parental strain. ${ }^{79}$ Apart from increased CA productivity, elimination of structurally similar 3S,5S clavams, which are relatively toxic metabolites, is highly desirable from a regulatory standpoint, and this also improved the efficiency of the downstream CA purification processes. Notably, the improved strain carrying the cvm1 and lat mutations showed increased titers in 11 and pilot 10001 fermentors, thus demonstrating that this increase in productivity is scalable. ${ }^{79}$ Given that the CA titer of the commercial strain is already several orders of magnitude higher than the wild-type titers, a $10 \%$ increase is quite significant. Thus, knowledge-based targeted manipulations can be applied to strains derived using conventional mutation and selection approaches, to further enhance strain productivity by eliminating competing pathways and structurally similar molecules, and leading to a more efficient overall process. The mechanism by which elimination of the cephamycin $\mathrm{C}$ pathway leads to an increase in CA remains to be established. However eliminating unrelated secondary metabolic pathways from the strain, and making general cellular resources available for the pathway of interest, could be explored further as a general approach to improve strain productivity.

The recent availability of genome sequences, the ability to construct genome scale in silico models to predict biochemical pathways and metabolic flux (flux-based models), and ability to refine such models with comparative 'omics' data (genomics, proteomics, transcriptomics and metabolomics) from strains with different phenotypes has opened up exciting possibilities to improve strains using newer approaches. An industrial high-producing S. clavuligerus strain obtained by iterative random mutagenesis and selection and producing CA at levels orders of magnitude higher than the wild type was subjected to transcriptome and genome-wide flux balance analysis to understand the changes that had occurred in this strain, which could explain the high-producing phenotype. Interestingly, the majority of the changes observed in the industrial strain were those that were also predicted and shown to be present in the recent wild-type derived strains constructed via directed knowledge-based strategies. ${ }^{80}$ For example, the industrial strain showed general overexpression of CA biosynthetic and regulatory genes, including $c c a R, c l a R$ and $a d p A$, whereas overexpression of the same genes in the wild-type strain led to enhanced CA production. Likewise, the changes in the expression levels of the glycerol metabolic genes in the industrial strain mimicked the phenotype associated with the gap-1 deletion mutation constructed in the wild-type background using rational approaches. These overlaps in the changes between strains obtained using random mutagenesis and screening and directed knowledge-based improvements have provided valuable insights into the key target genes and pathways responsible for overall strain productivity.

\section{FUTURE PERSPECTIVE}

CA (and Augmentin) continues to have a critical role in anti-infective therapy. Newer strategies are continually needed to improve Streptomyces strains for producing CA cost-effectively and to make this therapy affordable globally. Random mutagenesis and selection has had a critical role in developing strains for the commercial production of CA. This approach strictly depends on creating a pool of diverse mutants as randomly as possible, and identifying the best performing mutants from this pool, using appropriate screening or selection strategies. On the other hand, the knowledge-based targeted strain engineering approach can be used to construct strains in a very specific manner, by adding or deleting genes, or manipulating gene expression governing the desired phenotype, accordingly. The $S$. clavuligerus genome sequence is now available, ${ }^{81}$ and the cost of sequencing strains is decreasing rapidly. The ability to create genome-wide metabolic models (for example, a virtual cell) based on the genome sequence and the biochemical architecture of the strain, and the ability to feed the 'omics'-based data to build and validate such models, will further enhance our knowledge about CA biosynthesis and its regulation, and allow identification of additional targets for strain improvement in a more efficient manner. ${ }^{80,82}$ However, the knowledge-based targeted approach is still somewhat empirical because overproduction of metabolites is a complex phenotypic trait and involves a multitude of genes and pathways, and a desired phenotype cannot always be guaranteed by making defined changes one at a time. Perhaps it is more pertinent to have a combination of random mutagenesis and selection and knowledgebased approaches as a strategy for strain improvement. A recent example of this approach is the use of $c c a R$ promoter-reporter-based screen to identify S. clavuligerus strains, which have higher levels of $c c a R$ expression and higher CA production. ${ }^{83}$ Additionally, protoplast fusion-based genome shuffling techniques can speed up the generation of diverse mutant libraries for phenotypic screening and selections, and increase the probability of isolating the desired mutants. ${ }^{84-86}$ The key will be to design appropriate screening or selection strategies to identify the desired phenotypes. Subsequently, identification and rationalization of the critical mutations that lead to the desired phenotype in such mutants via genomics-based models, and then reverse engineering these mutations back into a suitable robust strain, which is more suitable for a commercial process, would be an attractive way forward.

\section{DEDICATION}

This article is dedicated to the memory of Professor Leo Charles Vining, who made significant contributions to the natural products chemistry and biosynthesis of secondary metabolites. As a former graduate student in Leo's laboratory at Dalhousie University, I have fond memories of our beloved Professor and mentor. Leo began his career as a traditional natural product chemist during the 1950s, a period that also belonged to the so called 'golden era of antibiotics'. Being in the prime of his scientific youth during this period, and engaged in making significant contributions in the area of natural product biosynthesis and regulation of antibiotic production, was perhaps very timely for Leo's career. Leo's passion included deciphering the various biosynthetic schemes, utilized by different bacteria, fungi and actinomycetes, for the production of diverse secondary 
metabolites, and understanding biochemical and physiological switches regulating the production of these metabolites. During the latter part of his career, he employed molecular biological tools toward understanding the molecular basis of antibiotic biosynthesis in actinomycetes. His career included several appointments, both in government institutes and academia, where he published 283 scientific articles and 2 books. Leo received several awards including the prestigious Charles Thom Award from the Society of Industrial Microbiology (SIM) and a Fellowship of the Royal Society of Canada for his outstanding contributions. Leo was a very smart and quick thinking scientist, an effective teacher and a very compassionate mentor. He had a very effective style of communication, which made him a very inspiring leader. What was amazing about Leo was that despite scaling so many heights in his career, he was very modest and down to earth in his approaches. He was always besides us, during good times celebrating exciting results, or during challenging times sharing his insights and expert opinions to create a path forward. Not surprising, his unassuming style and demeanor won him popularity and respect among students and peers, and his laboratory was one of the most sought after places to work in the field of Streptomyces, and represented a vibrant group of graduate students and post-doctoral fellows. Leo, you will be missed!!!

\section{ACKNOWLEDGEMENTS}

I thank Professor Arnold Demain and Professor Susan Jensen for providing helpful comments on the article.

1 Reading, C. \& Cole, M. Clavulanic acid: a $\beta$-lactamase-inhibiting $\beta$-lactam from Streptomyces clavuligerus. Antimicrob. Agents Chemother. 11, $852-857$ (1977).

2 Brown, A. G. et al. Naturally occurring $\beta$-lactamase inhibitors with antibacterial activity. J. Antibiotics 29, 668-669 (1976).

3 Labia, R., Barthelemy, M. \& Peduzzi, J. Molecular aspects of $\beta$-lactamase inhibition and inactivation by clavulanic acid: a review. Drugs Exp. Clin. Res. 11, 765-770 (1985).

4 Georgopapadakou, N. H. B-lactamase inhibitors: evolving compounds for evolving resistance targets. Exp. Opin. Investig. Drugs 13, 1307-1318 (2004)

5 Neu, H. C. \& Fu, K. P. Clavulanic acid, a novel inhibitor of $\beta$-lactamases. Antimicrob. Agents Chemother. 14, 650-655 (1978).

6 Demain, A. L. The Business of Biotechnology. Gen Publishing Inc. A Mary Ann Liebert Inc. Company 3, 269-283 (2007).

7 Elander, R. P. Industrial production of $\beta$-lactam antibiotics. Appl. Microbiol. Biotechnol. 61, 385-392 (2003).

8 Nagarajan, R. et al. $\beta$-lactam antibiotics from Streptomyces. J. Am. Chem. Soc. 93, 2308-2310 (1971).

9 Jensen, S. E. \& Paradkar, A. S. Biosynthesis and molecular genetics of clavulanic acid. Antonie van Leeuwenhoek 75, 125-133 (1999).

10 Baggaley, K. H., Brown, A. G. \& Schofield, C. J. Chemistry and biosynthesis of clavulanic acid and other clavams. Nat. Prod. Rep. 14, 309-333 (1997).

11 Janc, J. W., Egan, L. A. \& Townsend, C. A. Emerging evidence for a shared biosynthetic pathway among clavulanic acid and the structurally diverse clavam metabolites. Bioorg. Med. Chem. Lett. 3, 2313-2316 (1993).

12 Egan, L. A., Busby, R. W., Iwata-Reuyl, D. \& Townsend, C. A. Probable role of clavaminic acid as the terminal intermediate in the common pathway to clavulanic acid and the anti-podal clavam metabolites. J. Am. Chem. Soc. 119, 2348-2355 (1997).

13 Jensen, S. E. Biosynthesis of clavam metabolites. J. Ind. Microbiol. Biotechnol. 39, 1407-1419 (2012).

14 Kershaw, N. J., Caines, M. E., Sleeman, M. C. \& Schofield, C. J. The enzymology of clavam and carbapenem biosynthesis. Chem. Commun. 34, 4251-4263 (2005).

15 Baggaley, K. H., Elson, S. W., Nicholson, N. H. \& Sime, J. T. Studies on the biosynthesis of clavulanic acid. Part 4 . Synthetic routes to the monocyclic $\beta$-lactam precursor proclavaminic acid. J. Chem. Soc. Perkins Trans. I, 1513-1520 (1990).

16 Elson, S. W. \& Oliver, R. S. Studies on the biosynthesis of clavulanic acid. I. Incorporation of ${ }^{13} \mathrm{C}$-labelled precursors. J. Antibiot. 31, 586-592 (1978).

17 Elson, S. W., Oliver, R. S., Bycroft, B. W. \& Faruk, E. A. Studies on the biosynthesis of clavulanic acid. III. Incorporation of DL-[3,4-13C2]glutamic acid. J. Antibiot. 35, 81-86 (1982).

18 Jensen, S. E., Elder, K. J., Aidoo, K. A. \& Paradkar, A. S. Enzymes catalyzing the early steps of clavulanic acid biosynthesis are encoded by two sets of paralogous genes in Streptomyces clavuligerus. Antimicrob. Agents Chemother. 44, 720-726 (2000).
19 Perez-Redondo, R., Rodriguez-Garcia, A., Martin, J. F. \& Liras, P. Deletion of the pyc gene blocks clavulanic acid biosynthesis except in glycerol-containing medium: evidence for two different genes in formation of the C3 unit. J. Bacteriol. 181, 6922-6928 (1999)

20 Ward, J. M. \& Hodgson, J. E. The biosynthetic genes for clavulanic acid and cephamycin production occur as a 'super-cluster' in three Streptomyces. FEMS Microbiol. Lett. 110, 239-242 (1993).

21 Aidoo, K. A., Wong, A., Alexander, D. C., Rittammer, R. A. \& Jensen, S. E. Cloning, sequencing and disruption of a gene from Streptomyces clavuligerus involved in clavulanic acid biosynthesis. Gene 147, 41-46 (1994).

22 Paradkar, A. S., Aidoo, K. A., Wong, A. \& Jensen, S. E. Molecular analysis of a $\beta$-lactam resistance gene encoded within the cephamycin gene cluster of Streptomyces clavuligerus. J. Bacteriol. 178, 6266-6274 (1996)

23 Song, J. Y., Jensen, S. E. \& Lee, K. J. Clavulanic acid biosynthesis and genetic manipulation for its overproduction. Appl. Microbiol. Biotechnol. 88, 659-669 (2010).

24 Song, J. Y., Kim, E. S., Kim, D. W., Jensen, S. E. \& Lee, K. J. A gene located downstream of the clavulanic acid gene cluster in Streptomyces clavuligerus ATCC 27064 encodes a putative response regulator that affects clavulanic acid production. J. Ind. Microbiol. Biotechnol. 36, 301-311 (2009).

25 Khaleeli, N., Li, R. F. \& Townsend, C. A. Origin of the $\beta$-lactam carbons in clavulanic acid from an unusual thiamine pyrophosphate mediated reaction. J. Am. Chem. Soc. 121, 9223-9224 (1999).

26 Bachmann, B. O., Li, R. \& Townsend, C. A. B-lactam synthetase: a new biosynthetic enzyme. Proc. Natl Acad. Sci. USA 95, 9082-9086 (1998).

27 Hodgson, J. E. et al. Clavulanic acid biosynthesis in Streptomyces clavuligerus: gene cloning and characterization. Gene 166, 49-55 (1995).

$28 \mathrm{McNaughton}, \mathrm{H}$. J. et al. $\beta$-lactam synthetase: implications for $\beta$-lactamase evolution. Chem. Commun. (1998) 2325-2326 (1998).

29 Baldwin, J. E. et al. A substrate analog study on clavaminic acid synthetase: possible clues to the biosynthetic origin of proclavaminic acid. J. Chem. Soc. Chem. Commun. 1993, 500-502 (1993).

30 Marsh, E. N., Chang, M. D. \& Townsend, C. A. Two isozymes of clavaminate synthase central to clavulanic acid formation: cloning and sequencing of both genes from Streptomyces clavuligerus. Biochemistry 31, 12648-12657 (1992).

31 Salowe, S. P., Krol, W. J., Iwata-Reuyl, D. \& Townsend, C. A. Elucidation of the order of oxidations and identification of an intermediate in the multistep clavaminate synthase reaction. Biochemistry 30, 2281-2292 (1991).

$32 \mathrm{Wu}, \mathrm{T}$. K. et al. Identification, cloning, sequencing, and overexpression of the gene encoding proclavaminate amidino hydrolase and characterization of protein function in clavulanic acid biosynthesis. J. Bacteriol. 177, 3714-3720 (1995).

33 Salowe, S. P., Marsh, E. N. \& Townsend, C. A. Purification and characterization of clavaminate synthase from Streptomyces clavuligerus: an unusual oxidative enzyme in natural product biosynthesis. Biochemistry 29, 6499-6508 (1990).

34 Kershaw, N. J. et al. ORF6 from the clavulanic acid gene cluster of Streptomyces clavuligerus has ornithine acetyltransferase activity. Eur. J. Biochem. 269, 2052-2059 (2002).

35 de la Fuente, A., Martin, J. F., Rodriguez-Garcia, A. \& Liras, P. Two proteins with ornithine acetyltransferase activity show different functions in Streptomyces clavuligerus: Oat2 modulates clavulanic acid biosynthesis in response to arginine. J. Bacteriol. 186, 6501-6507 (2004).

36 Mosher, R. H., Paradkar, A. S., Anders, C., Barton, B. \& Jensen, S. E. Genes specific for the biosynthesis of clavam metabolites antipodal to clavulanic acid are clustered with the gene for clavaminate synthase 1 in Streptomyces clavuligerus. Antimicrob. Agents Chemother. 43, 1215-1224 (1999).

37 Tahlan, K. et al. 5S clavam biosynthetic genes are located in both the clavam and paralog gene clusters in Streptomyces clavuligerus. Chem. Biol. 14, 131-142 (2007).

38 Paradkar, A. S. \& Jensen, S. E. Functional analysis of the gene encoding the clavaminate synthase 2 isoenzyme involved in clavulanic acid biosynthesis in Streptomyces clavuligerus. J. Bacteriol. 177, 1307-1314 (1995).

39 Tahlan, K., Park, H. U., Wong, A., Beatty, P. H. \& Jensen, S. E. Two sets of paralogous genes encode the enzymes involved in the early stages of clavulanic acid and clavam metabolite biosynthesis in Streptomyces clavuligerus. Antimicrob. Agents Chemother. 48, 930-939 (2004)

40 Tahlan, K., Park, H. U. \& Jensen, S. E. Three unlinked gene clusters are involved in clavam metabolite biosynthesis in Streptomyces clavuligerus. Can. J. Microbiol. 50, 803-810 (2004)

41 Jensen, S. E., Wong, A., Griffin, A. \& Barton, B. Streptomyces clavuligerus has a second copy of the proclavaminate amidinohydrolase gene. Antimicrob. Agents Chemother. 48, 514-520 (2004).

42 Jensen, S. E. et al. Five additional genes are involved in clavulanic acid biosynthesis in Streptomyces clavuligerus. Antimicrob. Agents Chemother. 48, 192-202 (2004).

43 Arulanantham, H. et al. ORF17 from the clavulanic acid biosynthesis gene cluster catalyzes the ATP-dependent formation of N-glycyl-clavaminic acid. J. Biol. Chem. 281, 279-287 (2006).

44 Perez-Redondo, R., Rodriguez-Garcia, A., Martin, J. F. \& Liras, P. The claR gene of Streptomyces clavuligerus, encoding a LysR-type regulatory protein controlling clavulanic acid biosynthesis, is linked to the clavulanate-9-aldehyde reductase (car) gene. Gene 211, 311-321 (1998).

45 Nicholson, N. H. et al. Evidence that the immediate biosynthetic precursor of clavulanic acid is its N-aldehyde analog. J. Chem. Soc. Chem. Commun. 1994, 1281-1282 (1994). 
46 Mellado, E. et al. The clavulanic acid biosynthetic cluster of Streptomyces clavuligerus: genetic organization of the region upstream of the car gene. Microbiology 148, 1427-1438 (2002)

47 Lorenzana, L. M., Perez-Redondo, R., Santamarta, I., Martin, J. F. \& Liras, P. Two oligopeptide-permease-encoding genes in the clavulanic acid cluster of Streptomyces clavuligerus are essential for production of the $\beta$-lactamase inhibitor. J. Bacteriol. 186, 3431-3438 (2004)

48 Paradkar, A. S., Aidoo, K. A. \& Jensen, S. E. A pathway-specific transcriptional activator regulates late steps of clavulanic acid biosynthesis in Streptomyces clavuligerus. Mol. Microbiol. 27, 831-843 (1998).

$49 \mathrm{Li}, \mathrm{R}$., Khaleeli, N. \& Townsend, C. A. Expansion of the clavulanic acid gene cluster: identification and in vivo functional analysis of three new genes required for biosynthesis of clavulanic acid by Streptomyces clavuligerus. J. Bacteriol. 182, 4087-4095 (2000)

50 Iqbal, A. et al. Crystallographic and mass spectrometric analyses of a tandem GNAT protein from the clavulanic acid biosynthesis pathway. Proteins 78, 1398-1407 (2010).

$51 \mathrm{Bibb}$, M. J. Regulation of secondary metabolism in Streptomycetes. Curr. Opin Microbiol. 8, 208-215 (2005)

52 Liras, P., Gomez-Escribano, J. P. \& Santamarta, I. Regulatory mechanisms controlling antibiotic production in Streptomyces clavuligerus. J. Ind. Microbiol. Biotechnol. 35, 667-676 (2008).

53 Alexander, D. C. \& Jensen, S. E. Investigation of the Streptomyces clavuligerus cephamycin $\mathrm{C}$ gene cluster and its regulation by the CcaR protein. J. Bacteriol. 180, 4068-4079 (1998)

54 Perez-Llarena, F. J., Liras, P., Rodriguez-Garcia, A. \& Martin, J. F. A regulatory gene $(c c a R)$ required for cephamycin and clavulanic acid production in Streptomyces clavuligerus: amplification results in overproduction of both $\beta$-lactam compounds. J. Bacteriol. 179, 2053-2059 (1997).

55 Santamarta, I. et al. Characterization of DNA-binding sequences for CcaR in the cephamycin-clavulanic acid supercluster of Streptomyces clavuligerus. Mol. Microbiol. 81, 968-981 (2011)

56 Trepanier, N. K., Jensen, S. E., Alexander, D. C. \& Leskiw, B. K. The positive activator of cephamycin C and clavulanic acid production in Streptomyces clavuligerus is mistranslated in a bldA mutant. Microbiology 148, 643-656 (2002).

57 Bentley, S. D. et al. Complete genome sequence of the model Actinomycete Streptomyces coelicolor A3(2). Nature 417, 141-147 (2002)

58 Bignell, D. R., Tahlan, K., Colvin, K. R. Jensen, S. E. \& Leskiw, B. K. Expression of $c c a R$, encoding the positive activator of cephamycin $C$ and clavulanic acid production in Streptomyces clavuligerus, is dependent on bldG. Antimicrob. Agents Chemother 49, 1529-1541 (2005)

59 Bignell, D. R., Warawa, J. L., Strap, J. L., Chater, K. F. \& Leskiw, B. K. Study of the bldG locus suggests that an anti-anti-sigma factor and an anti-sigma factor may be involved in Streptomyces coelicolor antibiotic production and sporulation. Microbiology $146,2161-2173$ (2000).

60 Jnawali, H. N., Liou, K. \& Sohng, J. K. Role of sigma-factor (orf21) in clavulanic acid production in Streptomyces clavuligerus NRRL3585. Microbiol. Res. 166, 369-379 (2011).

61 Jnawali, H. N., Oh, T. J., Liou, K., Park, B. C. \& Sohng, J. K. A two-component regulatory system involved in clavulanic acid production. J. Antibiot. 61, 651-659 (2008).

62 Kinoshita, H., Tsuji, T., Ipposhi, H., Nihira, T. \& Yamada, Y. Characterization of binding sequences for butyrolactone autoregulator receptors in Streptomycetes. J. Bacteriol. $181,5075-5080$ (1999).

63 Onaka, H. \& Horinouchi, S. DNA-binding activity of the A-factor receptor protein and its recognition DNA sequences. Mol. Microbiol. 24, 991-1000 (1997).

$64 \mathrm{Jin}, \mathrm{W}$. et al. Cephamycin C production is regulated by relA and $r$ sh genes in Streptomyces clavuligerus ATCC27064. J. Biotechnol. 114, 81-87 (2004).

$65 \mathrm{Kim}, \mathrm{H}$. S. et al. Cloning and characterization of a gene encoding the gamma-butyrolactone autoregulator receptor from Streptomyces clavuligerus. Arch. Microbiol. 182, 44-50 (2004).
66 Hashimoto, K., Nihira, T. \& Yamada, Y. Distribution of virginiae butanolides and IM-2 in the genus. Streptomyces J. Ferment. Bioeng. 73, 61-65 (1992).

67 Santamarta, I., Perez-Redondo, R., Lorenzana, L. M. Martin, J. F. \& Liras, P. Different proteins bind to the butyrolactone receptor protein ARE sequence located upstream of the regulatory ccaR gene of Streptomyces clavuligerus. Mol. Microbiol. 56, 824-835 (2005).

68 Lopez-Garcia, M. T., Santamarta, I. \& Liras, P. Morphological differentiation and clavulanic acid formation are affected in a Streptomyces clavuligerus adpA-deleted mutant. Microbiology 156, 2354-2365 (2010).

69 Santamarta, I. et al. Connecting primary and secondary metabolism: AreB, an IcIR-like protein, binds the ARE $(c c a R)$ sequence of Streptomyces clavuligerus and modulates leucine biosynthesis and cephamycin $\mathrm{C}$ and clavulanic acid production. Mol. Mcrobiol. 66, 511-524 (2007).

70 Gomez-Escribano, J. P., Martin, J. F., Hesketh, A., Bibb, M. J. \& Liras, P. Streptomyces clavuligerus relA-null mutants overproduce clavulanic acid and cephamycin C: negative regulation of secondary metabolism by (p)ppGpp. Microbiology 154 744-755 (2008)

$71 \mathrm{Jin}, \mathrm{W}$. et al. Two relA/spoT homologous genes are involved in the morphological and physiological differentiation of Streptomyces clavuligerus. Microbiology 150 1485-1493 (2004)

72 Jiang, S. J., Yang, Y. Y. \& Wang, H. Q. Optimization of clavulanic acid fermentation Chi. J. Antibiot. 29, 335-337 (2004).

$73 \mathrm{Li}$, R. \& Townsend, C. A. Rational strain improvement for enhanced clavulanic acid production by genetic engineering of the glycolytic pathway in Streptomyces clavuligerus. Metab. Eng. 8, 240-252 (2006).

74 Banos, S., Perez-Redondo, R., Koekman, B. \& Liras, P. Glycerol utilization gene cluster in Streptomyces clavuligerus. Appl. Environ. Microbiol. 75, 2991-2995 (2009).

75 Jnawali, H. N., Yoo, J. C. \& Sohng, J. K. Improvement of clavulanic acid production in Streptomyces clavuligerus by genetic manipulation of structural biosynthesis genes. Biotechnol. Lett. 33, 1221-1226 (2011).

76 Song, J. Y., Kim, E. S., Kim, D. W., Jensen, S. E. \& Lee, K. J. Functional effects of increased copy number of the gene encoding proclavaminate amidino hydrolase on clavulanic acid production in Streptomyces clavuligerus ATCC 27064. J. Microbiol. Biotechnol. 18, 417-426 (2008).

77 Hung, T. V. et al. Enhancement of clavulanic acid by replicative and integrative expression of ccaR and cas2 in Streptomyces clavuligerus NRRL3585. J. Microbiol. Biotechnol. 17, 1538-1545 (2007).

78 Jnawali, H. N., Lee, H. C. \& Sohng, J. K. Enhancement of clavulanic acid production by expressing regulatory genes in gap gene deletion mutant of Streptomyces clavuligerus NRRL3585. J. Microbiol. Biotechnol. 20, 146-152 (2010).

79 Paradkar, A. S. et al. Applications of gene replacement technology to Streptomyces clavuligerus strain development for clavulanic acid production. Appl. Environ. Microbiol. 67, 2292-2297 (2001).

80 Medema, M. H. et al. Genome-wide gene expression changes in an industrial clavulanic acid overproduction strain of Streptomyces clavuligerus. Microb. Biotechnol. 4, 300-305 (2011)

81 Song, J. Y. et al. Draft genome sequence of Streptomyces clavuligerus NRRL 3585, a producer of diverse secondary metabolites. J. Bacteriol. 192, 6317-6318 (2010).

82 Medema, M. H., Alam, M. T., Breitling, R. \& Takano, E. The future of industria antibiotic production: from random mutagenesis to synthetic biology. Bioeng. Bugs 2 230-233 (2011)

83 Xiang, S. H. et al. Application of a double-reporter-guided mutant selection method to improve clavulanic acid production in Streptomyces clavuligerus. Metab. Eng. 11, 310-318 (2009).

84 Stephanopoulos, G. Metabolic engineering by genome shuffling. Nat. Biotechnol. 20, 666-668 (2002).

85 Zhang, Y. X. et al. Genome shuffling leads to rapid phenotypic improvement in bacteria. Nature 415, 644-646 (2002).

86 Gong, J., Zheng, H., Wu, Z., Chen, T. \& Zhao, X. Genome shuffling: Progress and applications for phenotype improvement. Biotechnol. Adv. 27, 996-1005 (2009). 